\title{
Proud lonely athletes: Using network centrality analysis to clarify the societal values of sports after the 2011 disaster in Japan
}

\author{
SASAKI Koh \\ Nagoya University, Nagoya, Japan \\ Takumi Yamamoto \\ National Defense Academy, Yokosuka, Japan \\ Yuichi Ueno \\ Ryutsu Keizai University, Ryugasaki, Japan \\ Takashi Katsuta \\ Japan Sport Council, Tokyo, Japan \\ Ichiro Kono \\ Japan Sport Council, Tokyo, Japan
}

\begin{abstract}
This research builds on our previous study, examining recognition of social management and observes the interpretation of benevolent sports activities following Japan's 2011 earthquake and tsunami. After the incident, most sports and recreational activities were suspended; however, various benevolent sports activities were arranged to benefit the damaged areas. This study investigates the recognised human value structure of sports in situation of great public anxiety by applying the life management concepts of time perspective and hope. Cluster analysis (Ward's method) was conducted to further explore the relationships structure in the values complex. The results regarding the terminal values study suggest the greatest recognition of 'hope for the future', 'family bonds', 'excellence', 'global concern' and 'life force'. Furthermore, the instrumental values study suggest greatest recognition of the 'ambitious', 'courageous', 'self-reliant', 'competent' and 'respectful' that indicate concern for inner motivation and survival capability. Network analysis (graph theory and centering resonance analysis) presents a holistic evaluation method for the positional functions of societal values and organization. This network centrality analysis clarifies the human values communicated by sports after the 2011 disaster. The comparison of national certified sports coaches, university students in sport science and university students in engineering reveals that athletes' lives are difficult and they experience loneliness while achieving victories and records. They feel pressurized to be the last man on the podium. Therefore, they might desire true friendship and family bonds with people sharing the same supreme desire, like fellow soldiers. However, if the loneliness is accompanied by some sense of societal pride, the combination could offer a positive social force for the future.
\end{abstract}

Keywords: human values, sports, network analysis

INTRODUCTION

Since the East Japan disaster on 11 March 2011, Japanese society has been at risk of losing collective hope for the future. This catastrophe caused much of the general public to refrain from all sports and recreational activities, even as various benevolent sports activities were 
immediately arranged to benefit the afflicted areas. Many national teams and professional leagues held charity matches, along with providing local support and relief goods and serving affected residents through personal networking and various other donation activities. The present study builds on our previous work [1], which examined the recognised human value structure provided by sports in this crisis situation by referring to the social management concepts of time perspective and hope.

The study of hope, as a social management tool, began in 2000 [2,3]. The purpose of this field of study is not to explore facile optimism but to consider the structure of positive and autonomic thinking and its role in contributing to community rebuilding. The study of human values is an approach designed to clarify the cognitive beliefs that operate in decision making and dealing with conflicts. Hope, a crucial human value, is recognised as a valuable motivator of life management activities in a crisis situation. The academic purpose of the present study is to identify the human value structure in sports and its social impact.

As people live in a society impacted by various complex values, they must recognise and prioritise these values within their own personal value system. This system has two dimensions: terminal values (end states of existence) and instrumental values (modes of conduct). This study adopted Rokeach's model [4] to operationalise these two dimensions. Rokeach's model includes terminal values such as 'pride in country', 'true friendship', 'inner harmony' and 'global concern' as attributed values as well as instrumental values such as 'broadminded' and 'forgiving' as concrete mentality and 'courageous' and 'honest' as life skills. Individuals' holistic cognitive value chain structures for sports activities, including benevolence, would be expected to describe the relationship between success in sports and survival in society. For a discussion on human values, the study of hope or time perspective as a life management approach and the study of terror management [5] as biological ontology could be effective. Drawing on these viewpoints, this study discusses the value chain of ontological commitment to society's realistic survival.

\section{METHODS}

Rokeach's model contains 18 terminal and 18 instrumental values and suggests that their prioritization will be affected by the particular context. For example, 'pride in country' and 'global concern' would tend to assume higher priority in a situation of international conflict. Thus, to adapt Rokeach's model to Japan's current social conditions, the authors made various alterations to Rokeach's set of items. In the category of terminal values, the authors changed 'national security' (protection from attack) to 'pride in country' (to represent citizens' willingness to work together for their country's good). 'Happiness' was changed to 'hope for the future' so as to represent an ontological commitment to improving people's existence rather than an absolute state. 'World beauty' (beauty of nature and arts) was changed to 'world beauty' (beauty of human beings and nature). 'An exciting life' was changed to 'life force', 'a comfortable life' to 'prosperity', 'a sense of accomplishment' to 'excellence', 'a world at peace' to 'global concern', 'salvation' to 'benevolence', 'mature love' to 'ideology/culture' and 'pleasure' to 'pure delight'. Among the instrumental values, 'obedient' (dutiful, respectful) was changed to 'respectful' (dutiful) to mean proactive rather than passive willingness.

The subjects included three groups of people: national certified sports coaches $(\mathrm{N}=99$ : male $=$ 63 , female $=36$, age $=28-60$ years, average age $=41$ years), university students in sport science $(\mathrm{N}=278$ : male $=155$, female $=123$, age $=18-21$ years, average age $=20$ years $)$ and university students in engineering $(\mathrm{N}=100$ : male $=73$, female $=27$, age $=17-20$ years, average age $=20$ years). The research was conducted in October and November 2014. Subjects 
received an explanation of the study's structure and of various sports-related benevolent activities such as donations, charity matches and voluntary disaster relief activities. Next, they were asked, 'What types of values do you recognise in Japanese benevolent sports activities that intended to help affected members of society after the 2011 disaster?' In response to this question, the participants were asked to rank the 18 terminal and 18 instrumental values. In the item ordering, the highest-ranked item was given 18 points, the second item 17 points and so on. Next, we performed cluster analysis (using Ward's method) to further explore the close relationships between the values within each cluster. To completely understand comprehensive human values and their role in people's lives, open-ended descriptions would be required; thus, the approach selected in the present study has obvious methodological limitations. However, the ranking of values and the order relationships can clarify the latent value structure.

Finally, a network analysis was performed to explore the centrality of the multi-values space in benevolent sports activities. Calculating centrality is a major focus of social networking because it indicates what persons (or, in this case, what values) occupy critical positions in a network. There are some centrality measures that are derived from the adjacency matrix and constitute different mathematical computations on the same underlying date.

Our previous study used degree centrality and eigenvector centrality that are most commonly used by network analysts. Eigenvector centrality (Cev) is directly dependent on the centrality values of its connected neighbours. A high centrality value of the neighbours should result in a high centrality for the vertex under consideration [6].

For a given graph $G:=(V, E)$ with $|V|$ vertices and $|E|$ edges, let $A=($ be the adjacency matrix, i.e. $=1$ if vertex is linked to vertex $t$, and the numbers will increase depending on the number of connections. $=0$ if there is no connections between the vertices. is a scalar.

In the present study, we used Katz centrality as a variant of eigenvector centrality, and we include the potential intimacy relationship within human values of co-operations. We assume $=0.06$, which means a one-eighteenth contributor's coefficient by total numbers of the terminal and instrumental values.

Furthermore, we apply a unique statistical approach to understand the difference between the groups, especially when verifying the similarity of the network centralities. The map layout was calculated using the Fruchterman-Reingold algorithm [7], which is a force-directed layout algorithm for constructing a graph to indicate the centralization of the multi-values' positioning. The purpose was to position the vertices of a graph in two- or three-dimensional space so that all the edges were of approximately equal length and with as few crossing edges as possible. The force-directed algorithms achieved this by assigning forces among the set of vertices and the set of edges. The forces were applied to the vertices, pulling them closer together or pushing them further apart. This was iteratively repeated until the system reached an equilibrium state. Results showed some contributors that mapped centrally in the graph by value positions. 


\section{Overall priority order \\ Terminal values}

\section{RESULTS AND DISCUSSION}

The priority order of terminal values was 'hope for future', 'family bonds (close companionship)', 'excellence (lasting contribution)', 'global concern', 'life force (zest for living)', 'freedom (independence, free choice)', 'self-respect (self-esteem)', 'pure delight', 'true friendship', 'equality (brotherhood, equal opportunity for all)', 'pride in country', 'inner harmony (freedom from inner conflict)', 'world beauty (beauty of human beings and nature)', 'benevolence', 'prosperity', 'wisdom (a mature understanding of life)', 'dignity', and 'ideology/culture' (see Figure 1).

'Hope for the future' maintained its first-place ranking from the previous study [1]. 'Family bonds' rose from the sixth place in the previous study to the second place in this one. 'Excellence', used in place of 'a sense of accomplishment', was third in this study and second in the previous study of sport-related values. These and other results are discussed in the cluster analysis below.

\section{Instrumental values}

The priority ranking order for instrumental values was 'ambitious (hard-working, aspiring)', 'courageous (standing up for your beliefs)', 'self-reliant (independent)', 'competent (capable, effective)', 'respectful (dutiful)', 'polite (courteous)', 'responsible (dependable)', 'honest (sincere, truthful)', 'loving (affectionate)', 'forgiving (willing to pardon others)', 'helpful (welfare for others)', 'light-hearted (cheerful, joyful)', 'imaginative (creative)', 'self-controlled (self-disciplined)', 'broadminded (open-minded)', 'clean (neat)', 'intellectual/logical (intelligent)' and 'consistent (logical)' (Figure 1). The top two values of 'ambitious' and 'courageous' were also ranked amongst the top four values in the previous study.

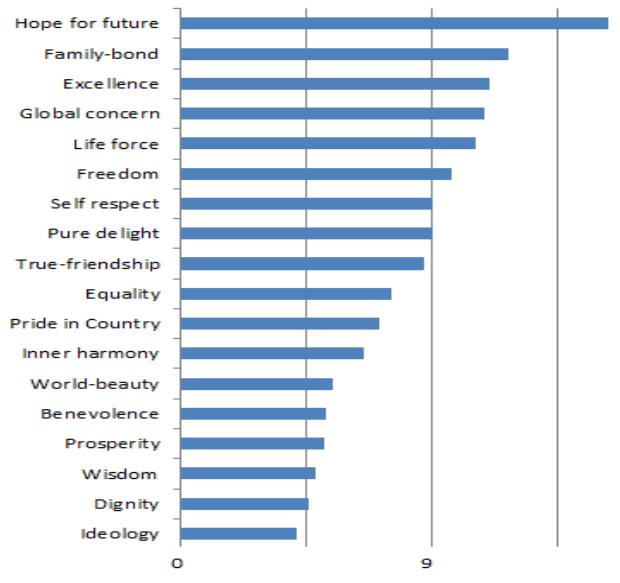

A; terminal values order

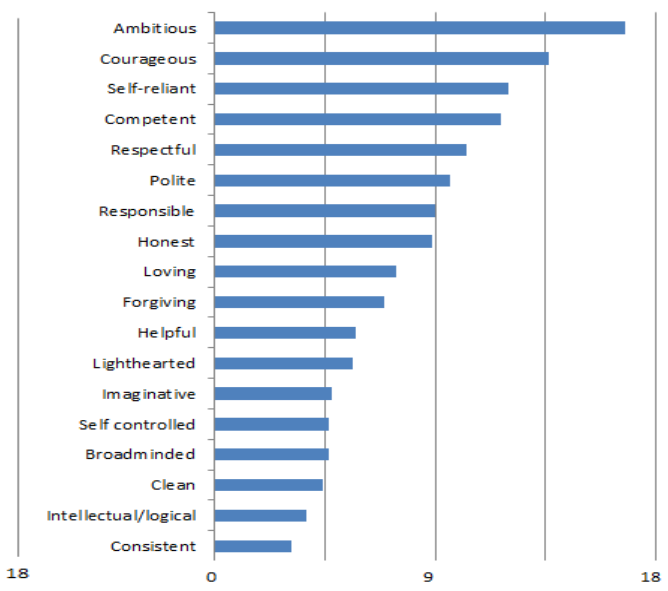

B: instrumental values order

Figure 1. Order of terminal (A) and instrumental (B) values assigned to benevolent sports activities after the 2011 disaster in Japan.

\section{Cluster analysis and network analysis National certified coaches}

We now discuss the three groups (national certified coaches, university students in sport science and university students in engineering) and the dimensions that they highlighted. The first group comprised the experienced athletic coaches and teachers. Their central cognitive societal terminal values (see Figure 2) were 'true friendship' (relationship), 'self-respect' and 
'inner harmony (freedom from inner conflict)'. Other values located near the central space were 'excellence (lasting contribution)', 'wisdom (a mature understanding of life)' and 'world beauty (human being and nature)'.

In response to the spreading sense of helplessness in society after the 2011 disaster, the coaches might perceive sports as strengthening human networking (true friendship) and mental control (inner harmony), promoting self-discipline (related to self-respect) and hard work and helping people to overcome social terror and anxiety and to appreciate the beauty of human nature. The coaches might support the younger generation not only with technical instruction but also in character building.

In the cluster analysis of the experienced coaches and teachers, we find two connections. First, true friendship and inner harmony were strongly connected with hope for the future. The coaches might believe that social intimacy has a role in encouraging hope for the future. Second, self-respect and excellence were strongly connected with global concern, suggesting a belief that dedication to sports has some relationship with global concerns. The other characteristics are the desire and centripetal force for belonging (pride in country, life force, wisdom).
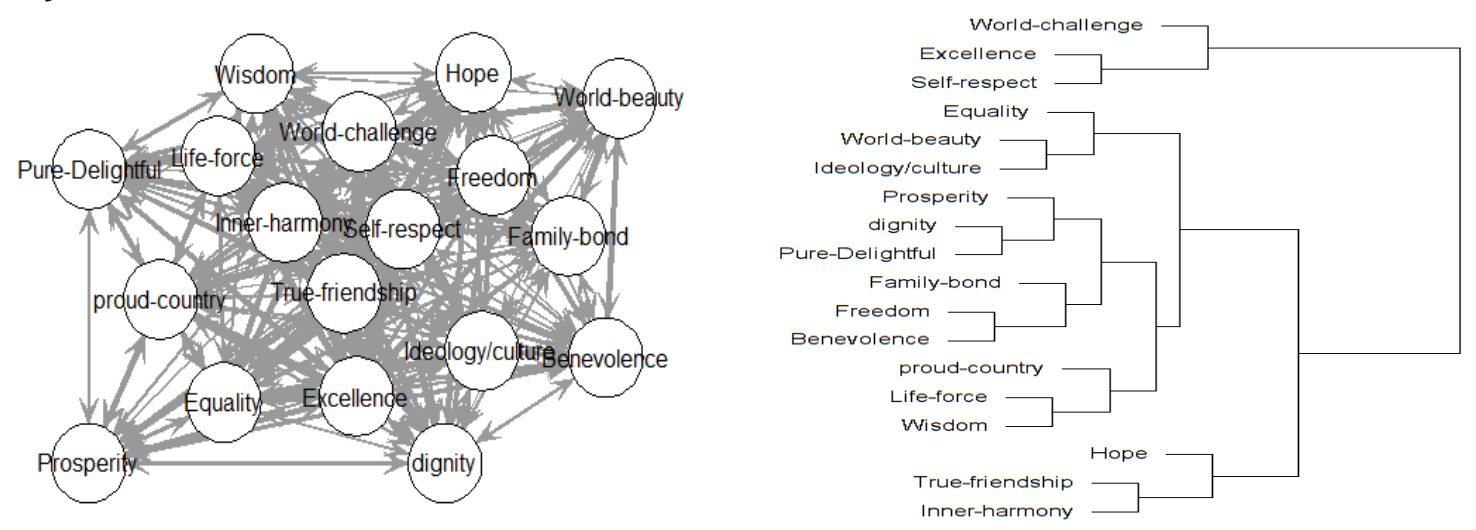

A: terminal values
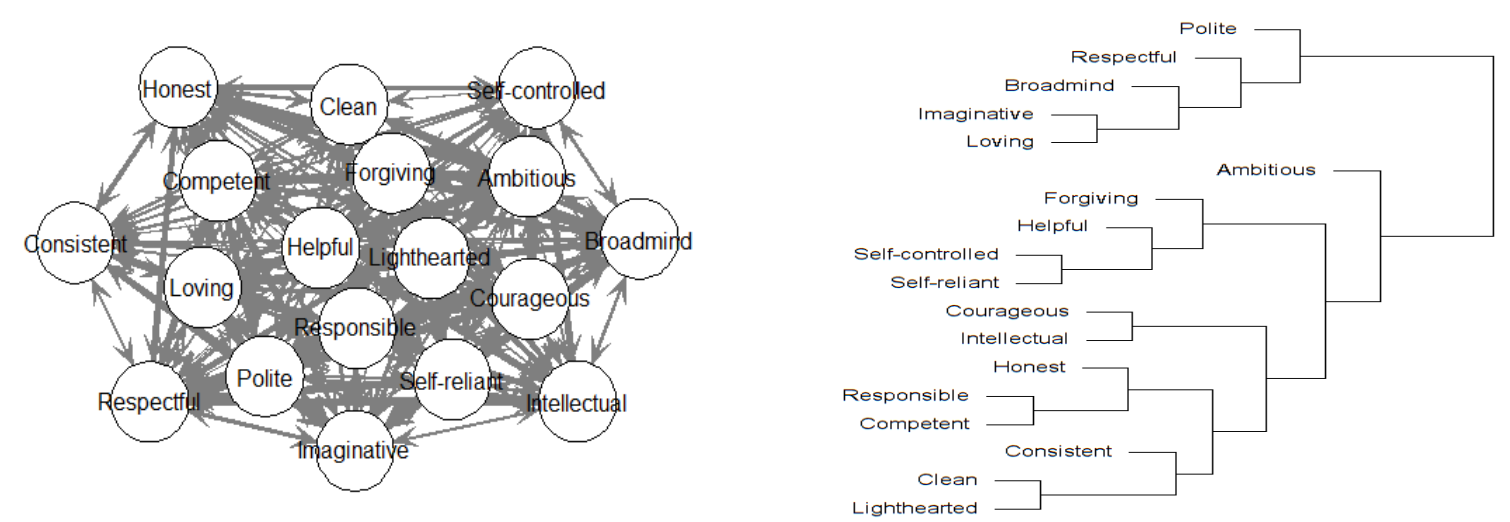

B: instrumental values

Figure 2. Mapping of societal terminal values of benevolent sports activities after the 2011 disaster by national certified coaches (A: terminal values; B: instrumental values; left: forcedirected placement (Katz centrality); right: cluster analysis).

These respondents' central cognitive societal instrumental values of sports were 'helpful (welfare for others)', 'light-hearted (cheerful, joyful)' and 'responsible (dependable)'. Other 
values located near the central space were 'polite (courteous)', 'forgiving (willing to pardon others)', 'ambitious (hard-working, aspiring)' and 'loving (affectionate)'. The coaches might regard interpersonal relationships as important for creating and re-enforcing societal networks. In cluster analysis, 'helpful' was closely related to 'forgiving', 'self-controlled' and 'self-reliant'; 'light-hearted' was closely related to 'clean' and 'consistent'. These clusters might show the importance of positive thinking in sports as a means of sustaining lasting dedication. These clusters expanding from 'helpful' and 'light-hearted' might suggest the altruism in sports as practical and essential senses.

\section{University students in sport science}

These students include not only athletes involved in national-level competitions but also some elite participants at the Olympic or World Cup level. Their central cognitive societal terminal values of sports (see Figure 3) were 'family bonds (close companionship)' and 'pure delight'. Other values located near the central space were 'life force (zest for living)', 'ideology/culture' and 'freedom (independence, free choice)'. Athletes might need close companionship and an experience of pure delight because they are relatively lonely in their dedicated preparation for high-level competitions. The answer of 'life force (zest for living)' might be suitable for the tough competitors. In the cluster analysis of the university students in sport science, 'family bond' was close to 'equality', 'life-force' and 'global concern'. These relationships might highlight the cooperative nature of team sports. 'Pure delight' was close to 'excellence', 'selfrespect' and 'inner harmony', perhaps emphasizing the importance of a positive view of oneself.

This group's central cognitive societal instrumental values of sports were 'broadminded (open-minded)' and 'imaginative (creative)'. The other value located nearest to the central space was 'intellectual/logical (intelligent)'. In the cluster analysis, 'broadminded' was close to 'forgiving'. The athletes might have similar characteristics to the coaches with regard to societal values because both groups attach greater importance to human skills than to technical skills.
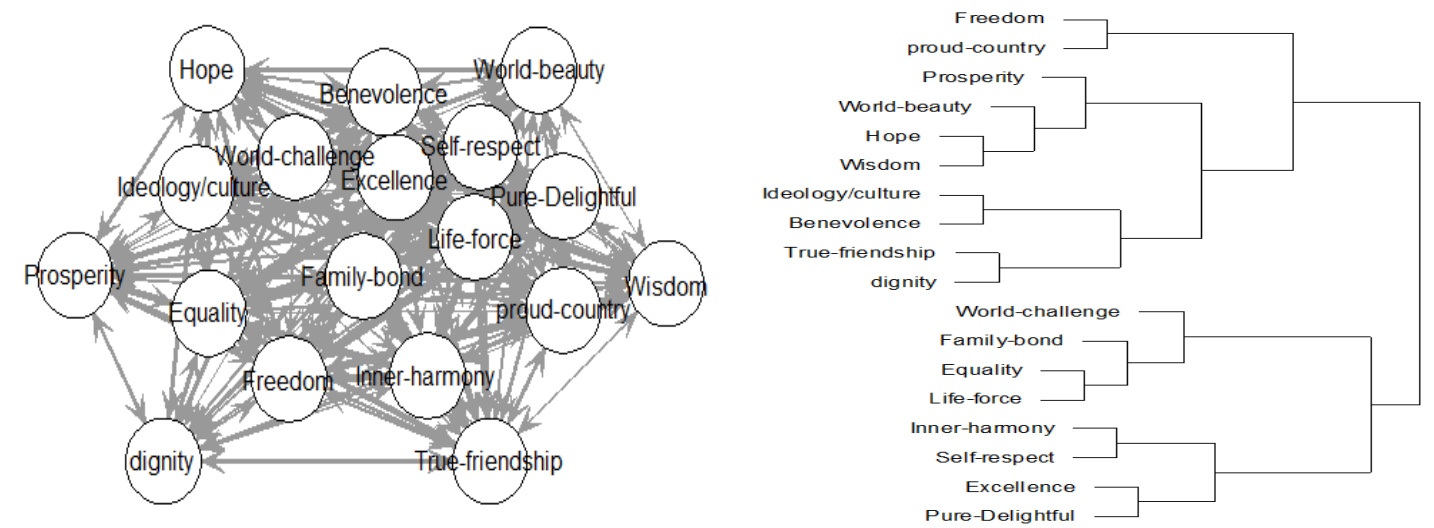

A: terminal values 

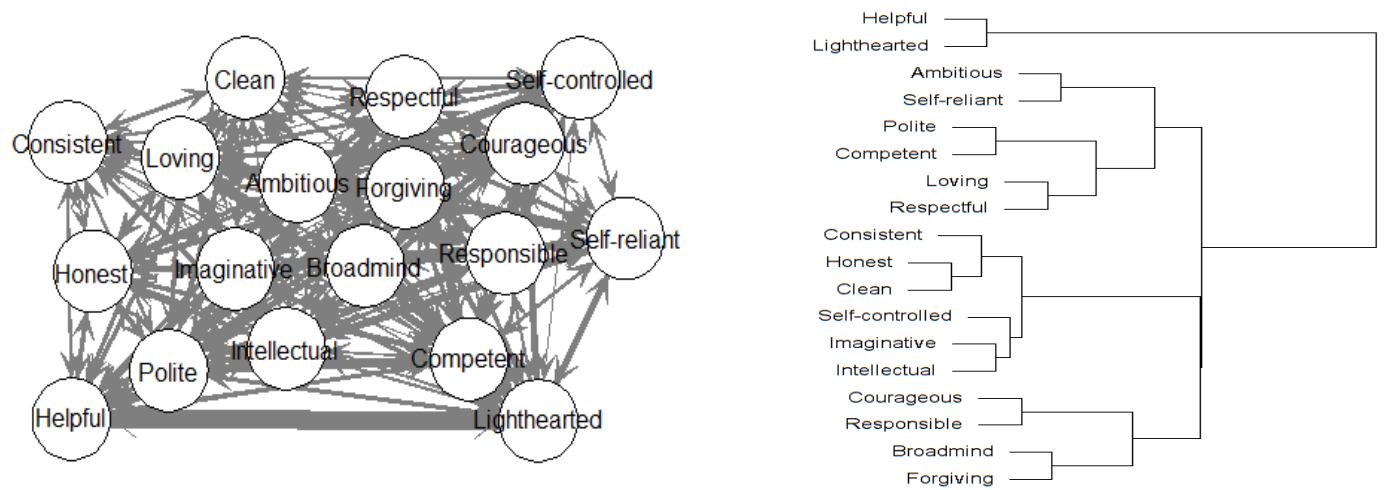

B: instrumental values

Figure 3. Mapping of societal terminal values of benevolent sports activities after the 2011 disaster by university students in sport science (A: terminal values; B: instrumental values; left: force-directed placement (Katz centrality); right: cluster analysis).

\section{University students in engineering}

These students are not coaches and athletes; i.e. they are not involved in sports. Thus, we should expect their answers to come from a different viewpoint. Their central cognitive societal terminal values of sports (see Figure 4) were 'equality (brotherhood, equal opportunity for all)' and 'prosperity'. Other values located near the central space were 'benevolence' and 'dignity'. 'Equality', 'benevolence' and 'dignity' would represent a realistic view of the values promoted by all levels of sport. On the other hand, 'prosperity' might refer to the sports that have ample resources. In the cluster analysis of these students' responses, 'equality' was close to 'prosperity', 'benevolence' was close to 'life force', and 'dignity' was close to 'wisdom'.

This group's central cognitive societal instrumental values of sports were 'consistent (logical)', 'self-controlled (self-disciplined)', 'honest (sincere, truthful)' and 'clean (neat)'. These respondents might regard sports achievement as the result of consistent, long-term effort. In the cluster analysis, the close relationships of values ('consistent' and 'intellectual', 'selfcontrolled' and 'ambitious', 'honest' and 'competent') might also reflect some key characteristics of sports.
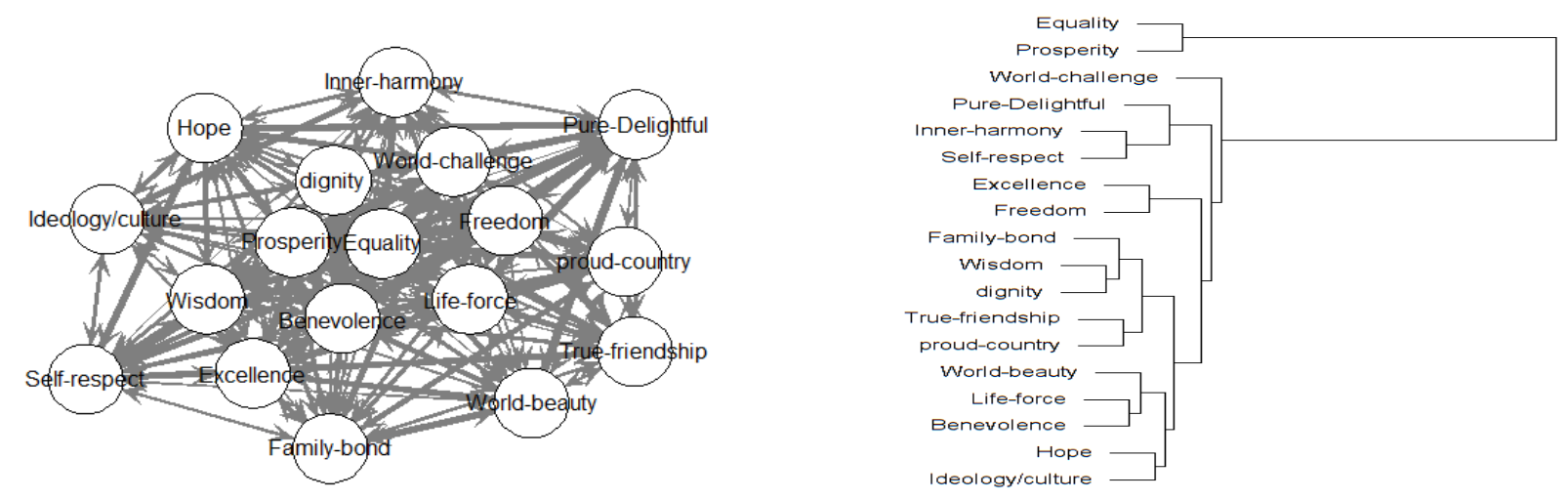

A: terminal values 

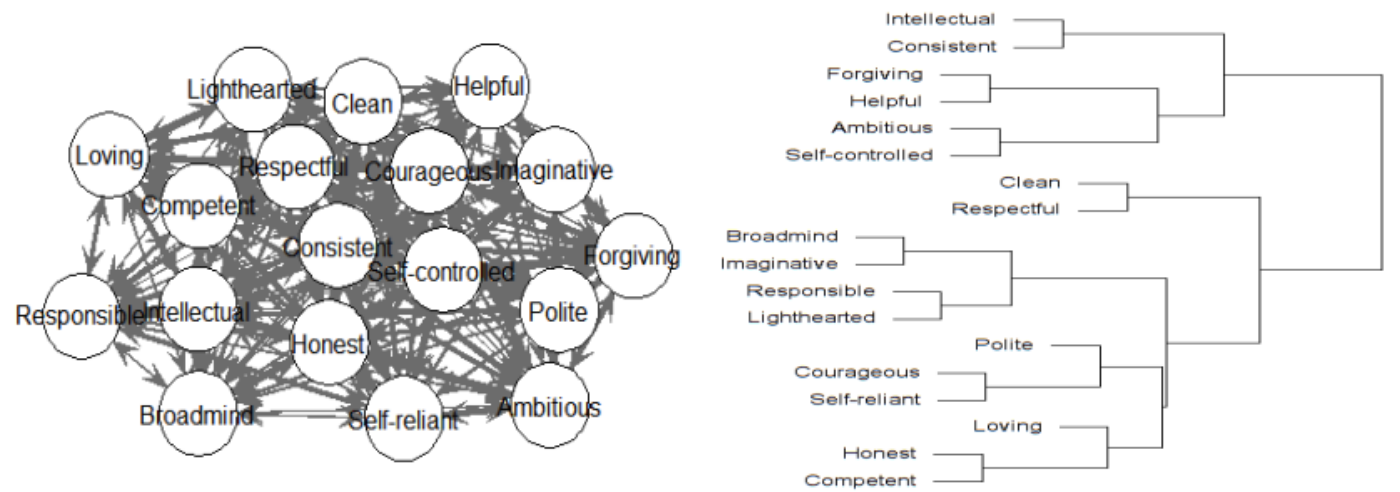

\section{B: instrumental values}

Figure 4. Mapping of societal terminal values of benevolent sports activities after the 2011 disaster by university students in engineering (non-sport students) (A: terminal values; B: instrumental values; left: force-directed placement (Katz centrality); right: cluster analysis).

\section{Hidden loneliness in athletes}

Network graph analysis can verify the difference or similarity between some graphs. The correspondence analysis, which is one of 'centering resonance analysis' methods, was examined to clarify the similarities and compare the network centrality (Figure 5). To maximise the relationship between row and column items, correspondence analysis sorts both rows and columns. As for the calculation result of the axial contribution ratio, the accumulated contribution ratio on the second axis is $100 \%$ (terminal values: $72.73 \%, 27.27 \%, 0.00 \%$; instrumental values: $65.81 \%, 34.19 \%, 0.00 \%$ ). The information on the graphed data was sufficiently representative.

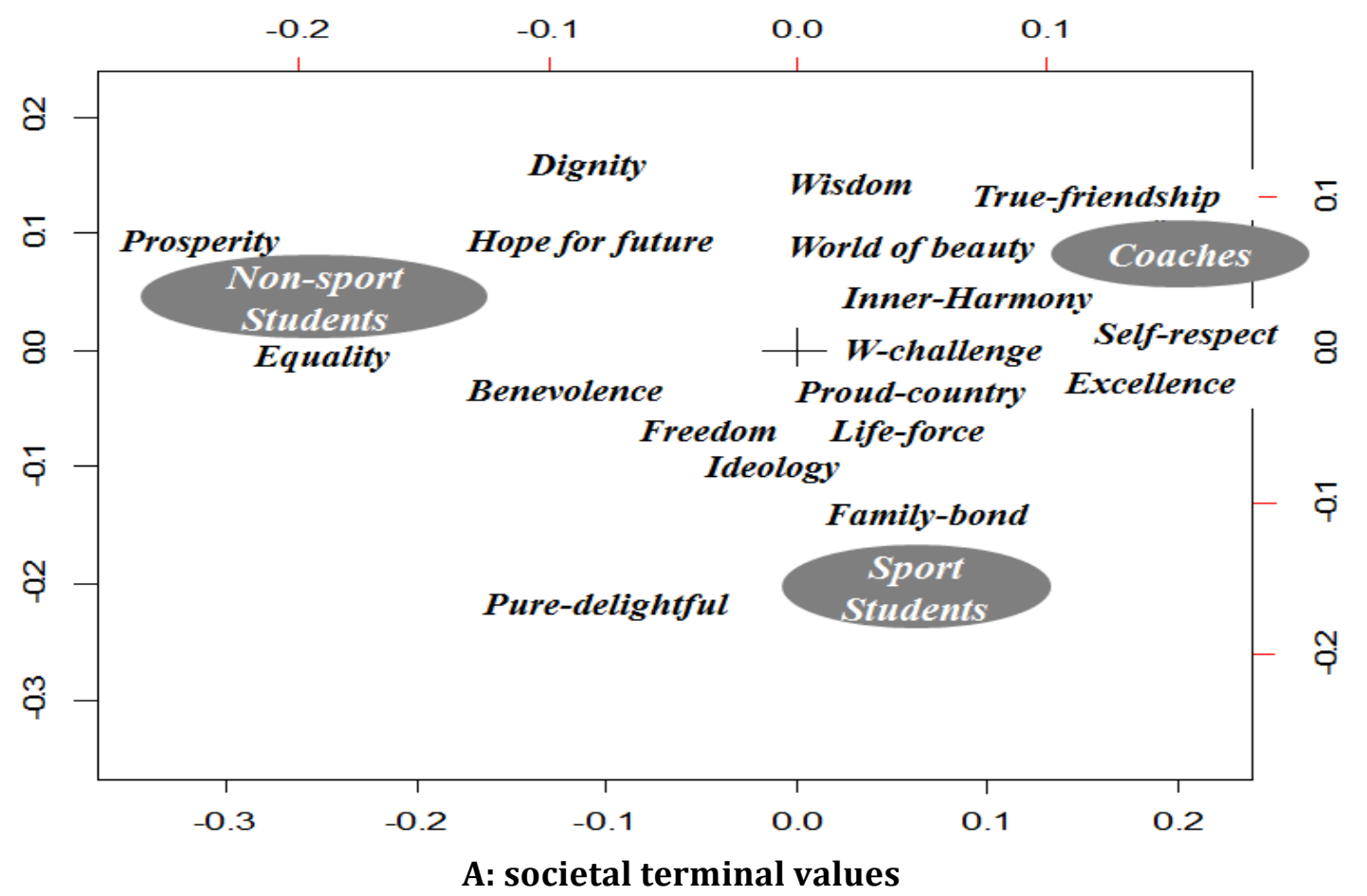




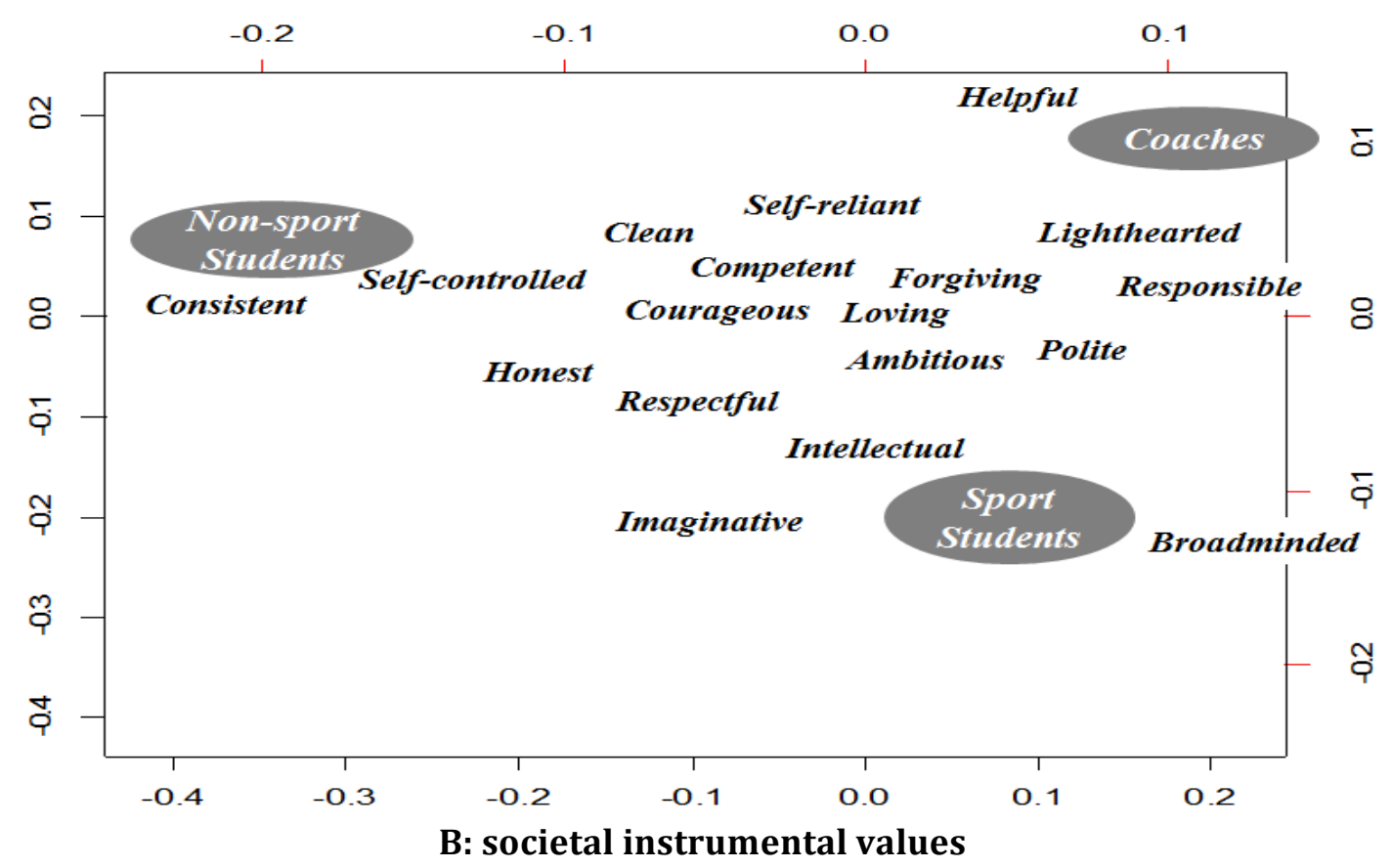

Figure 5. The space difference or similarity of the societal human values between coaches, elite sport students and non-sport students after the 2011 disaster in Japan as shown by centering resonance analysis (A: societal terminal values: B: societal instrumental values).

There might be very few athletes who could achieve the ideal societal terminal values (e.g. 'prosperity', 'equality') as reported by non-athlete students (university students in engineering). The societal values reported by the athletes (university students in sport science) who dedicate their lives to gruelling practice and competition were 'family bond' and 'pure delight'. National certified coaches also highlighted values concerned with human relations ('true friendship' and the indispensable 'self-respect') followed by technical values ('excellence'). These responses might suggest that athletes' lives are difficult with many lonely days to overcome on the path to victories and records. They feel very heavy pressure to be the last man standing on the podium. Therefore, the lonely athletes might desire true friendship and family bonds with people who share the same supreme desire, like fellow soldiers. Their central values in the graph might reflect an ambition to challenge the world as a representative of their country and a will to show benevolence. Societal loneliness has been proposed as one explanation of the spread of anxiety within our highly advanced information society in some sociological discussions (the 'lonely crowd', [8] 'habits of the heart' [9] and 'bowling alone' [10]). [11]. However, if the loneliness is accompanied by some sense of societal pride, the combination might have the potential to offer a positive social force for the future.

As for cognitive societal instrumental values, the engineering students reported 'consistent' and 'self-controlled', which are comparable ideal values. In contrast, the athletes might have emphasised 'broadminded' to remove the isolation of loneliness and 'imaginative' and 'intellectual/logical' to challenge the impossible dream. The coaches might report 'helpful', 'clean', 'responsible' and 'forgiving' as means of constructing positive human relationships.

In our previous study of hope after the 2011 disaster in Japan, we reported on how people regained hope to step into tomorrow, calling on their various societal human values and expending their emotional resources to invest in their own future. Furthermore, we reported 
that Japanese elite athletes might be motivated to take on the challenge of a long-term commitment to tough competition and might be regarded as the proud, lonely companions who express their proud country's will to recover from disaster. Reconstruction after a major disaster takes a long time, and at first people could not see the light at the end of the tunnel. Only the brave, who were willing to embrace the challenge, could move society towards a positive future.

Terror management theory provides one way of interpreting how people face the risks of life. Risk presents not only a negative, threatening dimension but also a more positive perspective, indicating that buffers against anxiety are provided not only by cultural and religious institutions but also by close human relationships [12]. Increasing the desire for relationships with others might act as a terror management mechanism.

Our prior study reported that the values recognised in sports might indicate common ontological commitments: 'Human existence could reflect a dynamic balance of both biological and ontological values, such that humankind lives with not only one value but also freely selected and compounded multi-values to adapt to an increasingly complex environment and society' [1]. Our findings regarding the societal human values expressed in sports by our lonely but proud athletes can be regarded as identifying one valuable aspect of Japan's social capital. Our country will host the 2019 Rugby World Cup and the 2020 Olympic and Paralympic Games. We hope that, as many people from around the world come together for these events, many values that can provide hope for the next generation will be produced and disseminated.

\section{ACKNOWLEDGEMENTS}

This study was partly supported by JSPS KAKENHI grant Number 24500736 (2011-2014).

\section{References}

K. Sasaki, K. Komatsu, T. Yamamoto, Y. Ueno, T. Katsuta, and I. Kono, Cognitive societal human values of sports: After the 2011 disaster of Japan, Social Sciences, 2(1), 1-6, 2013, DOI: 10.11648/j.ss.20130201.11.

Y. Genda, Social sciences of hope, Tokyo University Press, 2009.

M. Tsuzuki, Study of hope, Minerva Shobo, 2004.

M. Rokeach, The nature of human values, The Free Press, 1973.

D. L. Paulhus and P. D. Trapnell, Terror management theory: extended or overextended? Psychological Inquiry, 8(1), 40-43, 1997.

B. Junker and F. Schreiber, Analysis of biological networks, John Wiley \& Sons, Inc., P78, 2008.

T. M. J. Fruchterman and E. M. Reingold, Graph Drawing by Force-directed Placement, Software - Practice and Experience, 21(11), 1129-1164, 1991.

D. Riesman, lonely crowd, Yale University Press, 1950.

E. R. N. Bellah, Habits of the heart: Individualism and commitment in American life, University of California Press, 1985.

R. D. Putnam, Bowling alone, Simon and Schuster, 2001.

A. Portes, Downsides of social capital, Proceedings of the National Academy of Sciences of the United States of America, 111(52), 18407-08, 2014.

V. Florian, M. Mikulincer, and G. Hirschberger, The anxiety-buffering function of close relationships: Evidence that relationship commitment acts as a terror management mechanism, Journal of Personality and Social Psychology, $82(4), 527-542,2002$. 